\title{
Exercise-induced increase in brain-derived neurotrophic factor in human Parkinson's disease: a systematic review and meta- analysis
}

Mark A. Hirsch ${ }^{*}$, Erwin E. H. van Wegen ${ }^{2}$, Mark A. Newman ${ }^{1}$ and Patricia C. Heyn ${ }^{3}$

\begin{abstract}
Background: Animal models of exercise and Parkinson's disease (PD) have found that the physiologic use of exercise may interact with the neurodegenerative disease process, likely mediated by brain derived neurotrophic factor (BDNF). No reviews so far have assessed the methodologic quality of available intervention studies or have bundled the effect sizes of individual studies on exercise-induced effects on BDNF blood levels in human PD.
\end{abstract}

Research design and methods: We searched MEDLINE, EMBASE, Cochrane Library, PsycINFO and PubMed from inception to June 2017.

Results: Data aggregated from two randomized controlled trials and four pre-experimental studies with a total of 100 ambulatory patients with idiopathic PD (Hoehn/Yahr $\leq 3)$ found improvements in BDNF blood concentration levels in all 6 studies (two RCTs and 4 pre-experimental studies). Pooled BDNF level change scores from the 2 RCTs resulted in a significant homogeneous summary effect size (Standardized Mean Difference 2.06, 95\% Cl 1.36 to 2.76), and a significant heterogeneous SES for the motor part of the UPDRS-III examination (MD -5.53, 95\% Cl -10.42 to -0.64). Clinical improvements were noted in all studies using a variety of outcome measures.

Limitations: The evidence-base consists primarily of small studies with low to moderate methodological quality.

Conclusions: This review provides preliminary evidence for the effectiveness of physical exercise treatments for persons with PD on BDNF blood levels. Further research is needed.

Keywords: Brain derived neurotrophic factor, Exercise, Rehabilitation, Systematic review, Parkinson's disease

\section{Background}

Parkinson's disease (PD) is a complex, chronic, disabling neurodegenerative condition for which there is no cure [1]. The incidence of PD is expected to double in the next 15 years. The motor features of the disease include bradykinesia, rigidity, tremor, gait impairment and postural instability. Non-motor features include cognitive impairment, depression, sleep problems, osteoporosis, anxiety, fatigue and constipation. Increasingly, evidence supports efficacy of physical therapy and physical

\footnotetext{
* Correspondence: Mark.Hirsch@carolinashealthcare.org

${ }^{1}$ Carolinas Medical Center, Carolinas Rehabilitation, Department of Physical Medicine and Rehabilitation, 1100 Blythe Blvd, Charlotte, NC 28203, USA

Full list of author information is available at the end of the article
}

exercise interventions as adjunctive (i.e., helpful) to dopamine replacement therapy for control of motor symptoms and non-motor features, with improved quality of life for people at all stages of PD [2-9]. The physiologic effects of exercise may impact a number of plasticity-related events in PD brain including synaptogenesis, angiogenesis, and neurogenesis $[10,11]$.

In rodent PD models, physical exercise was found to interact with the neurodegenerative process [12-14], likely mediated by use-dependent expression of endogenous neurotrophic factors [5, 15-28]. The scientific evaluation of exercise induced changes in brain-derived neurotrophic factor (BDNF) concentration is emerging as a key research area in healthy adult populations [29-31] and in neurodegenerative populations (e.g., multiple sclerosis 
[32, 33]) (schizophrenia [34, 35]). Endogenous production of BDNF by voluntary exercise was shown in adult rats [36], and is purported to play a crucial role in neuroplastic effects of rehabilitation interventions of humans with neurodegenerative disease [28, 29, 37-40].

The physiologic mechanisms underlying exercise-induced BDNF changes are not well understood in PD but could include long-term potentiation and long-term depression mechanisms [41-43]. BDNF and exercise both promote survival and growth of neurons in pars compacta of substantia nigra and recovery of motor behavior [44]. In the 6-hydroxydopamine model of PD and exercise, blocking of BDNF receptors causes enhanced postlesion nigrostriatal dopaminergic cell loss, quantified as a reduction in the expression of tyrosine hydroxylase $(\mathrm{TH})$, a ratelimiting enzyme in dopamine biosynthesis [22, 45]. Additionally, BDNF may ameliorate neuronal dysfunction and neurodegeneration by modulating 1-methyl-4-phenylpyridinium (MPP+)-induced neurotoxicity [46], pathologic brain mitochondria function [47], or DNA repair by stimulating transcription factors such as CREB (cyclic AMP response element-binding protein) [48].

Recent reports by the Movement Disorder Society (MDS) Evidence-Based Medicine Panel on nonpharmacologic interventions for PD and the European Physiotherapy Guideline Development Group Panel recommended that future studies ought to focus on exerciseinduced neuroplasticity in humans with PD $[49,50]$. To the best of our knowledge, no reviews so far have assessed the methodologic quality of available intervention studies or have bundled the effect sizes of individual studies on exercise-induced changes in BDNF blood levels in human PD. The objective of this review was to systematically identify and appraise the evidence, methodological quality and clinical outcomes of intervention studies on the effects of physical exercise on endogenous production of BDNF in human PD, to bring such insights into the clinical context of rehabilitation for people living with PD.

\section{Method}

\section{Data sources and search strategy}

This study was conducted in accordance with the Preferred Reporting Items for Systematic Reviews and Meta-Analyses (PRISMA) statement [51]. An a priori protocol [52] was adhered to throughout the review process to minimize risk of bias. An electronic literature search was conducted independently by one of the authors $(\mathrm{PH})$ and a research assistant in the following databases: Medline (Ovid), PubMed (NLM), Embase (Embase.com), PsycINFO (Ovid), Physiotherapy Evidence Database (PeDro) and the Cochrane library (Wiley). We included the following key words (including MESH): Parkinson's, Parkinson's disease AND exercise, exercise training, physical activity, therapy, physical therapy, physical exercise, physical training, exercise- induced, exercise-enhanced AND human, people, person, individual, patient, older, elderly, AND neurotrophic factor, growth factor, brain derived neurotropic factor, neuroplasticity, plasticity, AND trial, intervention, training, treatment OR control, controlled, randomized.

\section{Criteria for inclusion}

We exclusively focused on studies evaluating the effects of exercise interventions on brain-derived neurotrophic factor in patients with PD. Studies were accepted when: 1) they used human participants with diagnosis of PD, 2) they used a prospective intervention design with or without a control group, 3) they contained physical exercise training or a physical exercise intervention component, 4) they assessed neurotrophic factor(s), 5) they were written in English, 6) they were published in a peerreviewed journal. Non-human studies, non-physical exercise trials, grey literature, studies using mixed populations, single case studies, studies not specific to PD, and studies without assessment of neurotrophic factors were excluded. The search was conducted up to June 2017.

\section{Review levels and data extraction}

Initial citation screening $(\mathrm{MH}, \mathrm{PH})$ was based on reviewing title and abstract (Level 1 Review) of all database search hits. A second round was implemented (Level 2 review) in which three independent reviewers $(\mathrm{EvW}, \mathrm{MN}, \mathrm{MH})$ analysed the full manuscripts and performed additional reference tracking. A total of 30 papers were imported into a widely used, web-based, production platform system for reviews (www.covidence.org). Disagreements were resolved in a consensus meeting (Fig. 1). Six manuscripts passed onto full data extraction (LEVEL 3 review in covidence.org). Data on study design, sample size and characteristics, exercise dosing, clinical outcome measures and laboratory results) were extracted manually by two independent data abstractors (MH, MN) and summarized in Table 1. Authors of relevant publications were contacted for data when post intervention means and/or SDs were not reported. When two or more randomized clinical trials were available reporting on the same outcomes, quantitative meta-analysis (i.e., pooling using Hedges' g) of the findings was performed using Cochrane methodology, in Review Manager 5.3 [53].

\section{Critical appraisal method}

Appraisal of individual study methodological quality was based on published quality assessment tools developed jointly by methodologists from NHLBI and Research Triangle Institute International (www.nhlbi.nih.gov/health-pro/ guidelines/in-develop/cardiovascular-risk-reduction/tools).

The NHLBI Appraisal tools (Additional file 1) includes items for evaluating the internal validity, descriptive criteria and statistical criteria of studies (Additional file 1). A quality rating ('good,' 'fair,' 'poor') was adapted from the review 


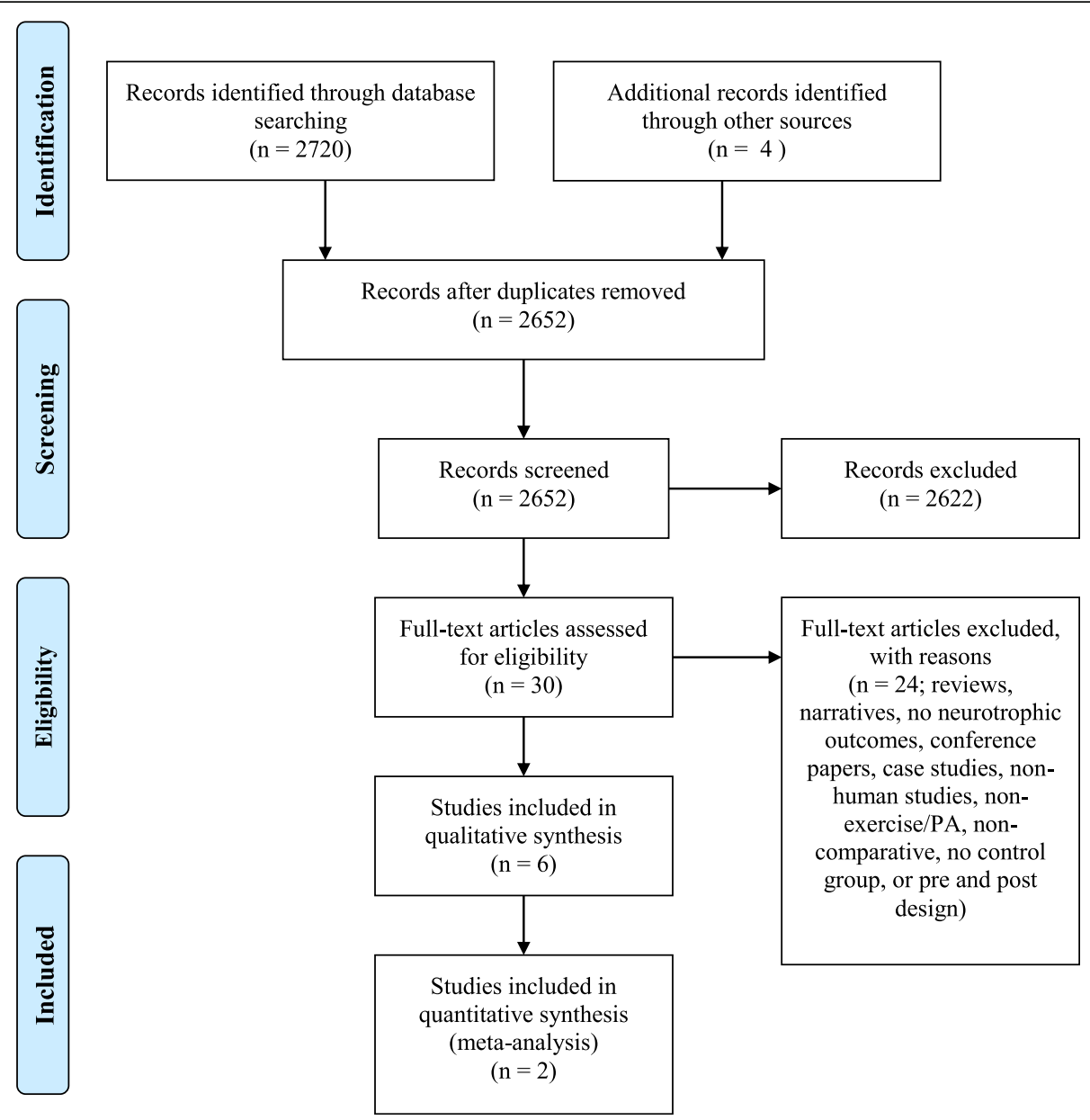

Fig. 1 PRISMA flow diagram

by Lim et al. [54]. Studies were considered to be of 'good' quality if at least $80 \%$ of the criteria were met; 'fair' quality when $51 \%$ to $79 \%$ of the criteria were met, and 'poor' quality when less than or equal to $50 \%$ of the criteria were met. Separate lists of methodological quality criteria were used for randomized controlled trials, uncontrolled pre-post studies and case control studies (Additional file 1).

Two evaluators $(\mathrm{MH}, \mathrm{MN})$ independently rated the methodological quality of the included studies using the NHLBI appraisal tools. Next, a kappa statistic was calculated for descriptive purposes and to investigate the agreement between the two evaluators on each appraisal tool (Additional file 1). The kappa values were interpreted using the criteria suggested by Tooth and Ottenbacher [55], <.40 poor agreement, .40 to .60 fair or moderate agreement, .60 to .80 good agreement, and $>.80$ perfect or excellent agreement. Additionally, we report the exact agreement among the two evaluators before disagreements about scoring were discussed (Additional file 1). Disagreements about scoring were resolved through discussion. If no consensus was reached, a third reviewer $(\mathrm{EvW})$ made the final decision.

\section{Effect size analysis}

For individual RCT's, we calculated the difference between the pre-to the post-intervention change scores for experimental and control groups. In case of MD-UPDRS-III, we used the mean difference (MD) between the change scores because the same outcome measure was assessed in the trials. For BDNF, reported as serum [56] and plasma levels [57], we used the standardized mean difference (SMD) based on Hedges' g by calculating the MD, divided by the average population standard deviation (SDi). The MD or SMD values of individual studies were averaged (pooled), resulting in a summary effect size (SES) with corresponding 95\% confidence interval (CI). Following Cohen [58] we classified effect sizes into small $(<0.2)$, medium $(0.2-$ $0.8)$, and large (>0.8). The $\mathrm{I}^{2}$ statistic was calculated to determine between-study variation [59]. In case of statistical heterogeneity $\left(\mathrm{I}^{2} \geq 50 \%\right.$, ) we applied a random-effects model. For $\mathrm{I}^{2}<50 \%$ a fixed-effect model was applied.

Two studies, one prospective study on exercise-induced changes in BDNF tyrosine receptor kinase signaling (BDNFTrkB) [60] and the prospective study by Zoladz and 


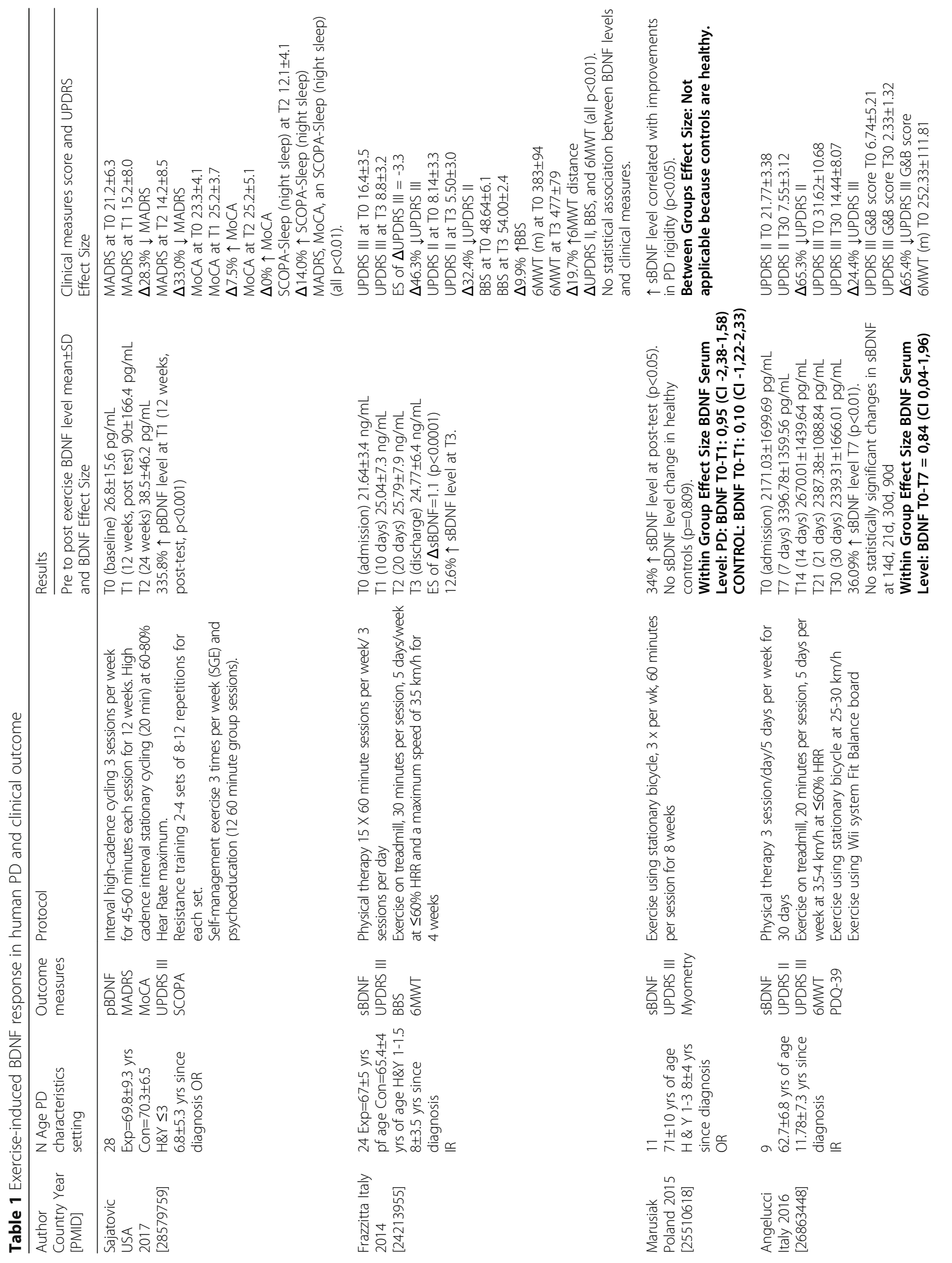




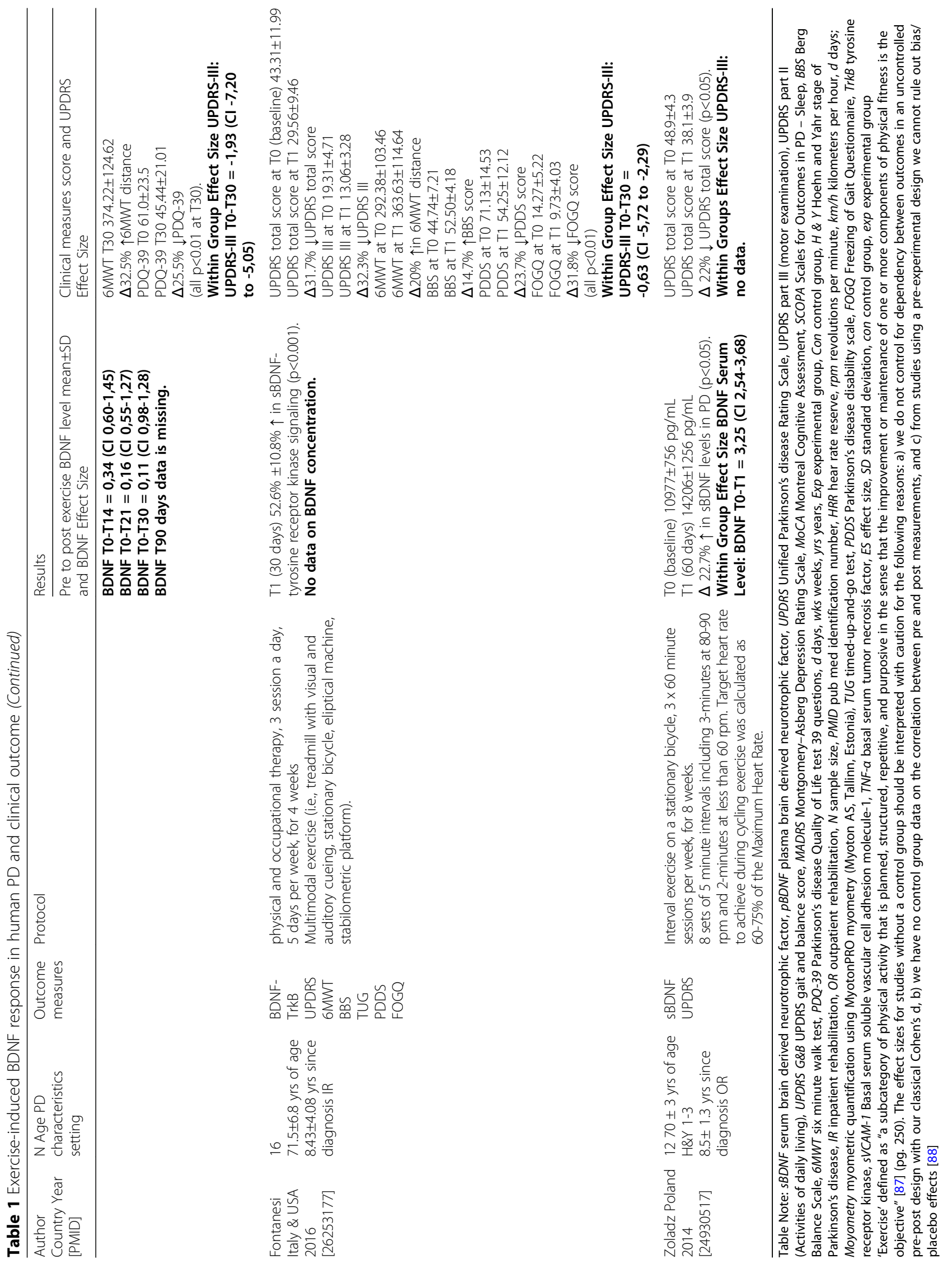


colleagues [61], were excluded from the BDNF level effect size analysis. The study by Fontanesi et al. [60] was excluded from the BDNF meta-analysis analysis because the authors did not report serum or plasma BDNF levels but instead reported BDNF tyrosine receptor kinase signaling. The study by Zoladz et al. [61] was excluded from the BDNF meta-analysis analysis because there was significant overlap in the study participant groups included in the paper by Zoladz et al. [61] and the study participants included in the paper by Marusiak et al. [62] (personal communication with Dr. Marusiak). The more recent candidate paper by Marusiak et al. [62] with the larger sample size (11 healthy controls and 11 patients with PD) was chosen for inclusion.

\section{Results}

\section{Summary of the literature}

The subject demographic characteristics, study design, exercise dosing, outcome measures and results are described in Table 1. A total of 100 participants contributed to the studies reported in this review. For the evaluation of physical exercise on BDNF levels, data were aggregated from two RCTs $[56,57]$ with a total of 52 ambulatory in- and outpatients with mild to moderate idiopathic PD severity, mean $7.0 \pm 1.5$ years after PD diagnosis and $68 \pm 5.6$ years (mean \pm standard deviation) of age at the time of study enrollment. BDNF concentration was assessed by enzymelinked immunosorbent assay (ELISA) using standardized procedures at the completion of the 28-day intervention in the study by Frazzitta et al. [56] and at the completion of the 90-day intervention in the study by Sajatovic et al. [57]. Percent change BDNF levels were reported from two pre-experimental studies (Marusiak et al. [62], Angelucci et al. [63] Table 1), with a total of 20 ambulatory in- and outpatients with mild to moderate idiopathic PD severity (Hoehn and Yahr stage $\leq 3$, range 1-3), 9.8 \pm 6.0 years (range 2-26 years) after PD diagnosis and $66.8 \pm 8.3$ years of age at the time of study enrollment. BDNF concentration was assessed by ELISA using standardized procedures at the completion of the 30-day intervention in the study by Angelucci et al. [63] and at the completion of the 60-day intervention in the study by Marusiak et al. [62].

Clinical outcomes data were aggregated from two RCTs $[56,57]$; and four pre-experimental studies [60-63] with a total of 100 ambulatory in- and outpatients with mild to moderate idiopathic PD severity (Hoehn and Yahr stage $\leq 3), 8.4 \pm 4.9$ years after PD diagnosis, $68.7 \pm 6.8$ years of age at the time of study enrollment. Methodological quality was scored for the six included studies [56, 57, 60-63] (Additional file 1).

Across all studies the participant characteristics were relatively homogenous in terms of years of age, PD stage, years since diagnosis, and ambulatory status. The studies were clinically homogenous with regards to including stationary cycling $[57,60-63]$ and administration of the MDS-UPDRS motor examination (Part III). Details about "on" or "off" state testing or training were not provided in the study by Fontanesi et al. [60] and Frazzitta et al. [56]. Physical exercise training, clinical outcome evaluations and BDNF testing procedures were conducted during the "on" phase in two studies [57, 63]. Zoladz et al. [61] and Marusiak et al. [62] administered outcome measures during the "off" phase. Differences among studies were noted regarding the use of elliptical machines [60], resistance training [57], physical therapy [56, 63], occupational therapy [60], treadmill training [56, 60, 63], Wii System Fit [63], and stabilometric platform [60]. All except one study reported exercise interventions being delivered by physiotherapists. Sajatovich et al. [57] used a Parkinson peer exercise leader to lead the exercise intervention. The setting for the interventions varied between laboratory [61, 62], hospital-based inand out-patient [56, 60,63] and fitness clinic-based [57]. The trial by Sajatovic et al. [57] was conducted in a community-based setting using a group versus an individual self-management program. In the study by Angelucci et al. [63] participants exercised in a group setting. None of the other studies reported details whether a group exercise intervention or an individual approach exercise intervention was used.

\section{BDNF levels}

BDNF levels assessed with laboratory measures were reported in 2 RCTs $[56,57](\mathrm{N}=52)$ and pooling resulted in a significant homogeneous SES (SMD 2.06, 95\% CI 1.36 to $2.76 ; \mathrm{Z}=5.77, P<.000001, \mathrm{I}^{2}=0 \%$, Fig. 2 ).

\section{Clinical outcomes}

MDS-UPDRS-III motor examination scores were reported in 2 RCTs $[56,57](N=52)$ and pooling resulted in a significant heterogeneous SES (MD -5.53, 95\% CI -10.42 to $-0.64 ; \mathrm{Z}=2.22, P=0.03, \mathrm{I}^{2}=94 \%$, Fig. 3). All studies noted statistically significant improvements for clinical outcome measures [56, 57, 60-63] (Table 1).

Few studies conducted statistical analysis between exercise-induced BDNF blood concentration and scores on clinical outcome measures. Marusiak et al. [62] found a statistically significant association between interval training induced increases in BDNF concentration and decrease in Parkinsonian rigidity. The study by Fontanesi et al. [60] found a statistically significant association between inpatient rehabilitation induced increases in TrkB signaling in the lymphocytes and improvement in MDSUPDRS total and MDS-UPDRS-II score. The trial by Frazzitta et al. [56] found no correlation between BDNF blood levels and the MDS-UPDRS motor examination score (Part III). 


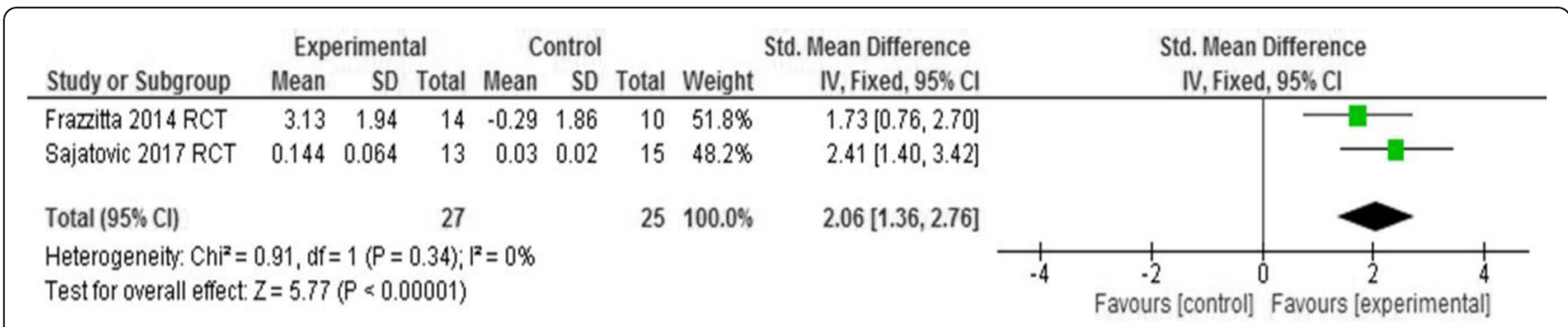

Fig. 2 Summary effect sizes for outcome of change in BDNF levels. Green squares indicate individual SES. Black colored diamond indicates the summary effect size; $R C T$ randomized clinical trial, SD standard deviation, Std standardized, CI Confidence Interval, $I^{2}$ statistic to determine heterogeneity, Z z-score

\section{Methodological quality}

A kappa statistic, which accounts for chance agreements between the two raters, was .62 for the trials by Frazzitta et al. [56] and Sajatovic et al. [57], .55 for the studies by Fontanesi et al. [60], Angelucci et al. [63] and Zoladz et al. [61], and .57 for the study by Marusiak et al. [62]. The percent agreement between the two raters was $72 \%$ to $77 \%$ (Additional file 1).

Strengths towards the internal validity of the studies included use of masked assessors, and administration of valid and reliable outcome measures. Four studies [56, $57,60,61]$ used assessors masked during the clinical outcomes testing, including the two randomized controlled trials [56, 57]. Five studies administered outcome measures that were valid, reliable and assessed consistently across all study participants [56, 57, 60-62]. Three studies reported loss to follow-up after baseline testing, which was less than 20\% [60-62].

Deficiencies included the lack of an a-priori justification for the sample size needed to detect an exercise-induced effect on BDNF levels [60-63], and lack of masking of the assessor conducting the BDNF assays [57, 60-63]. Additional deficiencies noted were lack of description of adherence to the intervention, short duration of training and short follow-up, lack of details about method used for participant recruitment into study protocol, failure to characterize the cognitive status of patients, failure to describe adverse events, and failure to recruit younger age patients.

Each 'deficiency' noted above represents an opportunity for future research and discovery. For example, age and physical exercise intensity may be a rate limiting factor in activity-dependent BDNF neuroplasticity. To address patient age, a future study on the effect of physical exercise on BDNF concentration could compare the response to exercise by age group because younger patients with PD typically display greater baseline physiologic reserve (e.g., $\mathrm{VO}_{2}$ Maximum) than older patients with $\mathrm{PD}$, and may be able to sustain physical exercise at higher physiologic intensities than older patients.

\section{Studies BDNF Assays Procedures}

BDNF assay procedures were reported by all studies. Sajatovic et al. [57] did not report if the blood sample collection was obtained in the morning or later during the day. Plasma samples of BDNF were assayed by using ELISA per manufacturer instructions (Quantikine ${ }^{\circ}$ ELISA Human BDNF Immunoassay; R\&D Systems, Minneapolis, MN, USA). Frazzitta et al. [56] did not report blood sample collection time but reported that serum BDNF concentrations were evaluated in a capture ELISA according to the protocol provided by the manufacturer without including

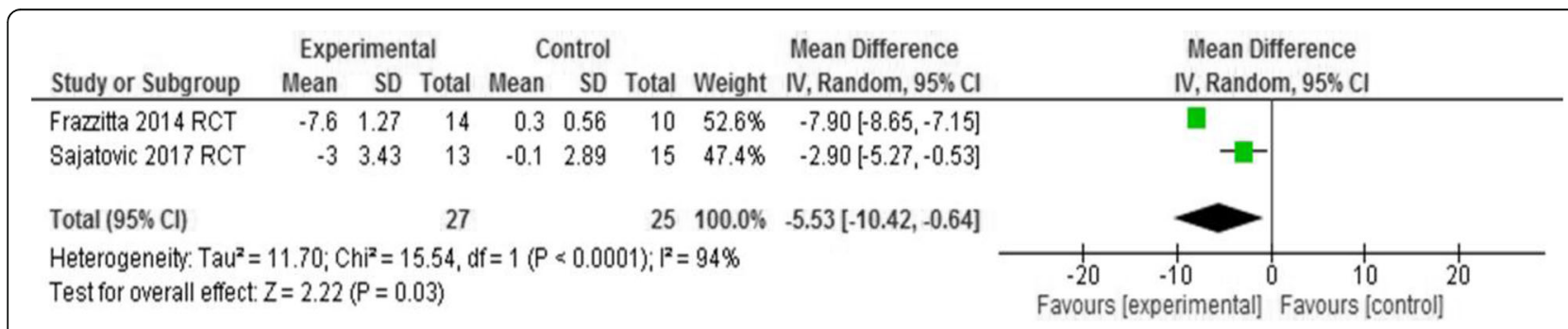

Fig. 3 Summary effect sizes for outcome of change in MDS-UPDRS motor score. Green squares indicate individual SES. Black colored diamond indicates the summary effect size; $R C T$ randomized clinical trial, SD standard deviation, Cl Confidence Interval, $I^{2}$ statistic to determine heterogeneity, Z z-score 
the kit manufacturer information. Marusiak et al. [62] and Zoladz et al. [61] assayed serum BDNF from morning blood samples with an ELISA Kit (Promega, Wallisellen, Switzerland) after appropriate dilution with Block and Sample solution (provided with the kit). Angelucci et al. [63] analyzed serum BDNF from blood samples that were obtained in the morning. Sandwich ELISA (R\&D Systems, USA; cat. No. DY248) was used for BDNF assays according to the manufacturer's instructions. Fontanesi [60] used morning blood samples for the BDNF assays by EDTA Western Blotting.

\section{Discussion}

The present systematic review and meta-analysis is the first to show aggregated evidence that physical exercise training increases BDNF blood levels in human PD. The main finding is that, in line with most pre-experimental studies that report significant results, pooling of the two available RCTs showed a significant SES in favour of physical exercise training for increasing BDNF levels. The BDNF results are paralleled by concomitant reductions in motor symptoms (UPDRS-scores), confirming possible effects on the dopaminergic pathways. Although our synthesis results support that controlled physical exercise training can have a positive impact on BDNF levels, and the number of publications in human PD is increasing, this review remains limited to a small number of studies that reported BDNF with few participants. Nevertheless, this is, to our knowledge, the first research synthesis study to evaluate the effects of controlled physical training trials on BDNF levels in human PD.

Our BDNF results are in line with prior human research in a variety of psychiatric conditions, and a recent review of neurotrophic factors in animal models of exercise and Parkinson's disease [42]. Studies utilizing psychiatric conditions have reported clinical improvements with increased serum BDNF levels following nonpharmacological approaches, including exercise [64, 65], computer-assisted cognitive enhancement in schizophrenia [35] and mindfulness clinical trials in bipolardisorder [66]. However, caution is warranted when extrapolating the results from psychiatric conditions to Parkinson's disease because clinical measures used in psychiatric conditions are different from the UPDRS.

Collectively, our meta-analysis found a SES of -5.53 point improvement on the motor examination part of the UPDRS, which is beyond the minimal clinically important difference [67]. Data from the included studies on exerciseinduced increases in BDNF blood concentration rarely correlated with clinical outcome measures [60, 62]. Studies are needed to determine the clinical relevance of exercise-induced increases in BDNF blood levels.

The most appropriate mode and intensity of exercise to achieve gains in BDNF concentrations in human PD remains controversial. For example, the 4-week intensive rehab training in the RCT of Frazzitta et al. [56] contained one hour of balance exercises and treadmill cue training, embedded in a daily three-hour general rehabilitation program but there is no information on duration of each subpart. In contrast, Sajatovic et al. [57] administered fastpaced, low-resistance cycling for 20 minutes followed by resistance training for 20 minutes using a progressive sequence of resistance band, 3 times a week for 12 weeks [57]. These are quite different modes and intensity of exercise training but apparently give similar, favorable results on blood BDNF concentration.

The kappa value of .55 to .57 , for the four uncontrolled studies and the kappa value of .62 for the two RCTs indicates fair to good agreement. The kappa value suggests that the raters accounted for $55 \%$ to $62 \%$ of the agreement over and above what would be expected by chance alone [55]. The percent exact agreement between the two raters - those instances for which both raters agreed that a study fulfills a methodological quality criteria - was $72 \%$ to $77 \%$, indicating raters agreed on over two-thirds of methodological quality items.

The evidence presented here is preliminary and does not address several important issues inherent to BDNF blood levels testing. BDNF has attracted increasing interest as potential biomarker to support the diagnosis or monitor the efficacy of therapies in brain disorders [64, 68]. Circulating BDNF levels can be measured in serum, plasma or whole blood. However, the use of BDNF as biomarker is limited by the probable poor reproducibility of results, likely due to the variety of methods used for sample collection and BDNF analysis, as well as the possible variations among performance for the different ELISA kits in term of intra-assay variation, inter-assay variation, detection range, and sensitivity [69]. In addition to the technical and methodological issues discussed here, several studies report that the socio-demographic determinants and other factors may affect serum levels of BDNF such as gender [70], age [71], body mass index [72], and disease status $[69,73]$. Several studies have suggested an interaction, with respect to circulating BDNF, between gender and age [70, 71, 74]. Meta-analyses and reviews of clinical studies based on the measurement of BDNF in whole blood, serum, or plasma have reported significantly lower BDNF levels at diagnosis in patients with mental illnesses [73-75]. These reviews however, highlighted severe discrepancies among studies, which even reported opposed results (increase versus decrease, or no change).

BDNF levels have also demonstrated to be affected by pharmacological treatments including antidepressant treatments $[64,75]$. Regarding description of pharmacologic treatments in the included studies, only the study by Frazzitta et al. [56] stated that, in order to minimize 
a possible polypharmacy effect, participants were enrolled if they were currently taking rasagiline monotherapy. In the studies by Fontanesi et al. [60] and Frazzitta et al. [56], patients remained on their pharmacotherapy regimen throughout the study duration. Sajatovic et al. [57] reported that patients were on a stable dose of levodopa medication throughout the trial. Regarding enrolment of participants on anti-depressant medication, the trial by Sajatovic et al. [57] included participants who were on a stable dose of antidepression medication for at least 1 month prior to trial enrolment. The trial by Frazzitta et al. [56] excluded patients who were on anti-depressant medication. The studies by Fontanesi et al. [60] Zoladz et al. [61] and Marusiak et al. [62] did not explicitly provide details about participants' anti-depression medication.

BDNF variability of response may relate, in part, to age, sex, medication and dietary factors, disease duration, cognitive status, air quality, or genetic factors [60, 76-82]. Studies have also shown differences in BDNF laboratory sample collection kits that may add an additional variability [83]. Concrete improvements to address variability in BDNF response may include adoption of repeated measures designs in which the sample is collected repeatedly from the same subject over time, pre and post training intervention (which would allow for within-subject comparisons), or use of surrogate markers of BDNF action (such as tyrosine receptor kinase signalling) as demonstrated in the forward thinking study by Fontanesi [60]. Additional improvements to decrease BDNF variability may include sampling from jugular vein catheters (instead of peripheral veins), or from saliva [82], directly after an exercise session.

\section{Limitations}

The main limitation of this review is the small number of papers that were available for inclusion. Although our results are based on a small number of studies, the participant characteristics were relatively homogenous in terms of years of age, Parkinson's disease stage, years since diagnosis, and ambulatory status. The studies were also homogenous in the adoption of cycling training mode, administration of the MDS-UPDRS and the pharmacologic characteristics of the participants [57, 60-63]. Our study is limited to the focus on one neurotrophic factor, exercise-induced changes in BDNF concentrations in human PD, and not other neurotrophic factors, which limits the generalizability to BDNF only. Although there are a number of potential opportunities for including BDNF as a clinical marker of brain health in PD [81], including outcome prediction [82], and/ or development of physical exercise treatment interventions [84], further studies and methodological evaluations need to take place to standardize BDNF measurement and evaluate its usefulness as a clinical marker of brain health in PD.
The Cochrane Library Guidelines do not recommend meta-analysis when the designs of the studies are too different, if the outcomes measured are not sufficiently similar, or if there are concerns about the quality of the studies, for an average result across the studies to be meaningful (for review, see http://www.cochranelibrary.com/about/aboutcochrane-systematic-reviews.html). Meta-analysis was a small part of the current review. We included all human studies, including non-randomized controlled studies (except case reports/single case studies as per exclusion criteria), covering the entire body of literature.

We emphasize that the small number of available studies is a limitation and further research is urgently needed to provide a realistic evaluation of the possible effects of exercise training on BDNF of PD patients. Prior international systematic reviews have evaluated effects of exercise training on BDNF and cognition (e.g., Alzheimer's) and usually these synthesis reports also have had a small number of studies (between 6-8) [85].

We propose that strengths of the current preliminary meta-analysis and systematic review include: a) the manuscript addresses a clinically important, understudied area of neurorestorative rehabilitation research, and b) the results challenge and seek to shift current research and clinical practice paradigms by extending novel theoretical concepts of physiologic use of exercise on neuroplasticity in ageing human brain to humans living with Parkinson's disease.

\section{Conclusions}

In summary, the presented results provide preliminary evidence of an exercise-induced increase in BDNF blood levels in human PD. Further high-quality, rigorously conducted randomized clinical trials of physical exercise effect on BDNF blood levels are needed to show robustness of the presented optimistic trend and to determine the neuroplastic mechanisms (for review, see [86]) that link BDNF blood levels, physical exercise, and functional outcomes in PD.

\section{Additional file}

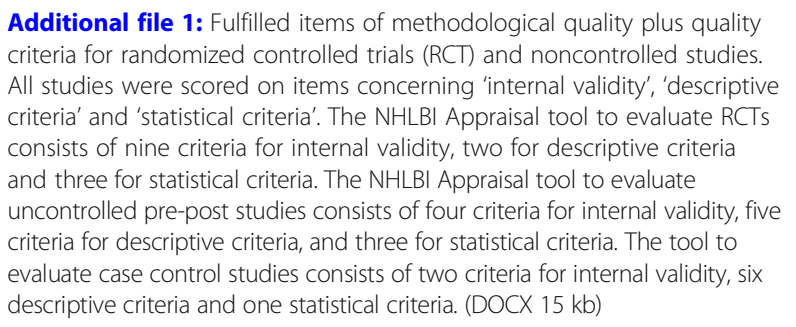

Additional file 1: Fulfilled items of methodological quality plus quality criteria for randomized controlled trials (RCT) and noncontrolled studies. All studies were scored on items concerning 'internal validity', 'descriptive criteria' and 'statistical criteria'. The NHLBI Appraisal tool to evaluate RCTs consists of nine criteria for internal validity, two for descriptive criteria and three for statistical criteria. The NHLBI Appraisal tool to evaluate uncontrolled pre-post studies consists of four criteria for internal validity, five criteria for descriptive criteria, and three for statistical criteria. The tool to evaluate case control studies consists of two criteria for internal validity, six descriptive criteria and one statistical criteria. (DOCX $15 \mathrm{~kb}$ )

\section{Abbreviations}

PD: Parkinson's disease; BDNF: brain-derived neurotrophic factor; TH: tyrosine hydroxylase; MPP+: 1-methyl-4-phenylpyridinium; CREB: cyclic AMP response element-binding protein; MDS: Movement Disorder Society; PRISMA: Preferred 
Reporting Items for Systematic Reviews and Meta-Analyses; MD: mean difference; SMD: standardized mean difference; SDi: population standard deviation; SES: summary effect size; BDNF-TrkB: brain-derived neurotrophic factor tyrosine receptor kinase signaling; ELISA: enzyme-linked immunosorbent assay

\section{Acknowledgements}

We thank Drs Marusiak, Sajatovic and Angelucci for providing the mean and standard deviation BDNF levels for the data in their papers. We also thank C. Gurnsey for her contribution to the data collection. We are happy to provide the data in aggregate form. We thank the reviewers for helpful comments.

\section{Funding}

None.

\section{Availability of data and materials}

Not applicable.

\section{Authors' contributions}

MAH conceived the study. All authors contributed equally to the intellectual content. PH assisted with a priori protocol development and with the data abstraction forms. EvW performed the statistical analysis. All authors approved the final version of the manuscript.

\section{Ethics approval and consent to participate}

Not applicable.

\section{Consent for publication}

Not applicable.

\section{Competing Interests}

The authors declare that they have no competing interests.

\begin{abstract}
Author details
${ }^{1}$ Carolinas Medical Center, Carolinas Rehabilitation, Department of Physical Medicine and Rehabilitation, 1100 Blythe Blvd, Charlotte, NC 28203, USA. 2Department of Rehabilitation Medicine, Amsterdam Movement Sciences/ Amsterdam Neurosciences, VU University Medical Center, PO Box 7057, 1007 Amsterdam, MB, The Netherlands. ${ }^{3}$ Department of Physical Medicine and Rehabilitation, Anschutz Medical Campus, University of Colorado, Denver, USA.
\end{abstract}

Received: 3 January 2018 Accepted: 28 February 2018 Published online: 20 March 2018

\section{References}

1. Kalia LV, Lang AE. Parkinson's disease. Lancet. 2015;386:896-912.

2. Cascaes da Silva F, lop Rda R, Domingos dos Santos P, Aguiar Bezerra de Melo LM, Barbosa Gutierres Filho PJ, da Silva R. Effects of Physical-ExerciseBased Rehabilitation Programs on the Quality of Life of Patients With Parkinson's Disease: A Systematic Review of Randomized Controlled Trials. J Aging Phys Act. 2016;24:484-96.

3. Lauze M, Daneault JF, Duval C. The Effects of Physical Activity in Parkinson's Disease: A Review. J Parkinsons Dis. 2016;6:685-98.

4. Munneke M, Nijkrake MJ, Keus SH, Kwakkel G, Berendse HW, Roos RA, Borm GF, Adang EM, Overeem S, Bloem BR. Efficacy of community-based physiotherapy networks for patients with Parkinson's disease: a clusterrandomised trial. Lancet Neurol. 2010:9:46-54.

5. Petzinger GM, Fisher BE, McEwen S, Beeler JA, Walsh JP, Jakowec MW. Exercise-enhanced neuroplasticity targeting motor and cognitive circuitry in Parkinson's disease. Lancet Neurol. 2013;12:716-26.

6. Ridgel AL, Walter BL, Tatsuoka C, Walter EM, Colon-Zimmermann K, Welter E, Sajatovic M. Enhanced Exercise Therapy in Parkinson's disease: A comparative effectiveness trial. J Sci Med Sport. 2016;19:12-7.

7. Saltychev M, Barlund E, Paltamaa J, Katajapuu N, Laimi K. Progressive resistance training in Parkinson's disease: a systematic review and metaanalysis. BMJ Open. 2016;6:e008756.

8. Schenkman M, Hall DA, Baron AE, Schwartz RS, Mettler P, Kohrt WM. Exercise for people in early- or mid-stage Parkinson disease: a 16-month randomized controlled trial. Phys Ther. 2012;92:1395-410.

9. Tomlinson $\mathrm{CL}$, Herd CP, Clarke CE, Meek C, Patel S, Stowe R, Deane KH, Shah L, Sackley CM, Wheatley K, Ives N. Physiotherapy for Parkinson's disease: a comparison of techniques. Cochrane Database Syst Rev. 2014: Cd002815.

10. Ahlskog JE. Does vigorous exercise have a neuroprotective effect in Parkinson disease. Neurology. 2011;77:288-94.

11. Hirsch MA, Farley BG. Exercise, neuroplasticity and Parkinson's disease. Eur J Phys Rehabil Med. 2009;45(2):215-29.

12. Fisher BE, Petzinger GM, Nixon K, Hogg E, Bremmer S, Meshul CK, Jakowec MW. Exercise-induced behavioral recovery and neuroplasticity in the 1methyl-4-phenyl-1,2,3,6-tetrahydropyridine-lesioned mouse basal ganglia. J Neurosci Res. 2004;77:378-90.

13. Petzinger GM, Walsh JP, Akopian G, Hogg E, Abernathy A, Arevalo P, Turnquist $P$, Vuckovic M, Fisher BE, Togasaki DM, Jakowec MW. Effects of treadmill exercise on dopaminergic transmission in the 1-methyl-4-phenyl1,2,3,6-tetrahydropyridine-lesioned mouse model of basal ganglia injury. J Neurosci. 2007;27:5291-300.

14. Tajiri N, Yasuhara T, Shingo T, Kondo A, Yuan W, Kadota T, Wang F, Baba T, Tayra JT, Morimoto T, et al. Exercise exerts neuroprotective effects on Parkinson's disease model of rats. Brain Res. 2010;1310:200-7.

15. Alexi T, Hefti F. Neurotrophin- $4 / 5$ selectively protects nigral calbindincontaining neurons in rats with medial forebrain bundle transections. Neuroscience. 1996;72:911-21.

16. Cohen AD, Tillerson JL, Smith AD, Schallert T, Zigmond MJ. Neuroprotective effects of prior limb use in 6-hydroxydopamine-treated rats: possible role of GDNF. J Neurochem. 2003:85:299-305.

17. Daviaud N, Garbayo E, Sindji L, Martinez-Serrano A, Schiller PC, Montero-Menei CN. Survival, differentiation, and neuroprotective mechanisms of human stem cells complexed with neurotrophin-3-releasing pharmacologically active microcarriers in an ex vivo model of Parkinson's disease. Stem Cells Transl Med. 2015:4:670-84

18. Gu S, Huang H, Bi J, Yao Y, Wen T. Combined treatment of neurotrophin-3 gene and neural stem cells is ameliorative to behavior recovery of Parkinson's disease rat model. Brain Res. 2009;1257:1-9.

19. Jiang P, Dang RL, Li HD, Zhang LH, Zhu WY, Xue Y, Tang MM. The impacts of swimming exercise on hippocampal expression of neurotrophic factors in rats exposed to chronic unpredictable mild stress. Evid Based Complement Alternat Med. 2014;2014:729827.

20. Jung SY, Kim DY, Yune TY, Shin DH, Baek SB, Kim CJ. Treadmill exercise reduces spinal cord injury-induced apoptosis by activating the PI3K/Akt pathway in rats. Exp Ther Med. 2014;7:587-93.

21. Koo HM, Lee SM, Kim MH. Spontaneous Wheel Running Exercise Induces Brain Recovery via Neurotrophin-3 Expression Following Experimental Traumatic Brain Injury in Rats. J Phys Ther Sci. 2013;25:1103-7.

22. Real CC, Ferreira AF, Chaves-Kirsten GP, Torrao AS, Pires RS, Britto LR. BDNF receptor blockade hinders the beneficial effects of exercise in a rat model of Parkinson's disease. Neuroscience. 2013;237:118-29.

23. Sauer $H$, Wong V, Bjorklund A. Brain-derived neurotrophic factor and neurotrophin-4/5 modify neurotransmitter-related gene expression in the 6hydroxydopamine-lesioned rat striatum. Neuroscience. 1995;65:927-33.

24. Sharma NK, Ryals JM, Gajewski BJ, Wright DE. Aerobic exercise alters analgesia and neurotrophin-3 synthesis in an animal model of chronic widespread pain. Phys Ther. 2010;90:714-25.

25. Woodlee MT, Schallert T. The interplay between behavior and neurodegeneration in rat models of Parkinson's disease and stroke. Restor Neurol Neurosci. 2004;22:153-61.

26. Wu SY, Wang TF, Yu L, Jen CJ, Chuang JI, Wu FS, Wu CW, Kuo YM. Running exercise protects the substantia nigra dopaminergic neurons against inflammation-induced degeneration via the activation of BDNF signaling pathway. Brain Behav Immun. 2011;25:135-46.

27. Ying Z, Roy RR, Edgerton VR, Gomez-Pinilla F. Exercise restores levels of neurotrophins and synaptic plasticity following spinal cord injury. Exp Neurol. 2005;193:411-9.

28. Zigmond MJ, Exercise SRJ. is it a neuroprotective and if so, how does it work? Parkinsonism Relat Disord. 2014;20(Suppl 1):S123-7.

29. Knaepen K, Goekint M, Heyman EM, Meeusen R. Neuroplasticity - exerciseinduced response of peripheral brain-derived neurotrophic factor: a systematic review of experimental studies in human subjects. Sports Med. 2010;40:765-801.

30. Szuhany KL, Bugatti M, Otto MW. A meta-analytic review of the effects of exercise on brain-derived neurotrophic factor. J Psychiatr Res. 2015;60:56-64.

31. Vaynman S, Gomez-Pinilla F. License to run: exercise impacts functional plasticity in the intact and injured central nervous system by using neurotrophins. Neurorehabil Neural Repair. 2005;19:283-95. 
32. Castellano V, White LJ. Serum brain-derived neurotrophic factor response to aerobic exercise in multiple sclerosis. J Neurol Sci. 2008;269:85-91.

33. Gold SM, Schulz KH, Hartmann S, Mladek M, Lang UE, Hellweg R, Reer R, Braumann KM, Heesen C. Basal serum levels and reactivity of nerve growth factor and brain-derived neurotrophic factor to standardized acute exercise in multiple sclerosis and controls. J Neuroimmunol. 2003;138:99-105.

34. Green MJ, Matheson SL, Shepherd A, Weickert CS, Carr VJ. Brain-derived neurotrophic factor levels in schizophrenia: a systematic review with metaanalysis. Mol Psychiatry. 2011;16:960-72.

35. Vinogradov S, Fisher M, Holland C, Shelly W, Wolkowitz O, Mellon SH. Is serum brain-derived neurotrophic factor a biomarker for cognitive enhancement in schizophrenia. Biol Psychiatry. 2009;66:549-53.

36. Gomez-Pinilla F, Zhuang Y, Feng J, Ying Z, Fan G. Exercise impacts brainderived neurotrophic factor plasticity by engaging mechanisms of epigenetic regulation. Eur J Neurosci. 2011;33:383-90.

37. Ahlskog JE, Geda YE, Graff-Radford NR, Petersen RC. Physical exercise as a preventive or disease-modifying treatment of dementia and brain aging. Mayo Clin Proc. 2011;86:876-84.

38. Murer MG, Yan Q, Raisman-Vozari R. Brain-derived neurotrophic factor in the control human brain, and in Alzheimer's disease and Parkinson's disease. Prog Neurobiol. 2001;63:71-124.

39. Neeper SA, Gomez-Pinilla F, Choi J, Cotman C. Exercise and brain neurotrophins. Nature. 1995;373:109.

40. Zuccato C, Cattaneo E. Brain-derived neurotrophic factor in neurodegenerative diseases. Nat Rev Neurol. 2009;5:311-22.

41. Campos C, Rocha NB, Lattari E, Paes F, Nardi AE, Machado S. Exerciseinduced neuroprotective effects on neurodegenerative diseases: the key role of trophic factors. Expert Rev Neurother. 2016;16:723-34.

42. da Silva PG, Domingues DD, de Carvalho LA, Allodi S, Correa CL. Neurotrophic factors in Parkinson's disease are regulated by exercise: Evidence-based practice. J Neurol Sci. 2016;363:5-15.

43. Hirsch MA, Hirsch HVB. The Adaptable Brain: Biology of Social Neuroplasticity. Topics in Geriatric Rehabilitation. 2014;30:2-7.

44. Razgado-Hernandez LF, Espadas-Alvarez AJ, Reyna-Velazquez P, SierraSanchez A, Anaya-Martinez V, Jimenez-Estrada I, Bannon MJ, Martinez-Fong $D$, Aceves-Ruiz J. The transfection of BDNF to dopamine neurons potentiates the effect of dopamine D3 receptor agonist recovering the striatal innervation, dendritic spines and motor behavior in an aged rat model of Parkinson's disease. PLoS One. 2015;10:e0117391.

45. Fumagalli F, Racagni G, Riva MA. Shedding light into the role of BDNF in the pharmacotherapy of Parkinson's disease. Pharmacogenomics J. 2006;6:95-104.

46. Nam JH, Leem E, Jeon MT, Jeong KH, Park JW, Jung UJ, Kholodilov N, Burke RE, Jin BK, Kim SR. Induction of GDNF and BDNF by hRheb(S16H) transduction of SNpc neurons: neuroprotective mechanisms of hRheb(S16H) in a model of Parkinson's disease. Mol Neurobiol. 2015;51:487-99.

47. Markham A, Bains R, Franklin P, Spedding M. Changes in mitochondrial function are pivotal in neurodegenerative and psychiatric disorders: how important is BDNF? Br J Pharmacol. 2014;171:2206-29.

48. Yang JL, Lin YT, Chuang PC, Bohr VA, Mattson MP. BDNF and exercise enhance neuronal DNA repair by stimulating CREB-mediated production of apurinic/apyrimidinic endonuclease 1. Neuromolecular Med. 2014;16:161-74

49. Fox SH, Katzenschlager R, Lim SY, Ravina B, Seppi K, Coelho M, Poewe W, Rascol O, Goetz CG, Sampaio C. The Movement Disorder Society EvidenceBased Medicine Review Update: Treatments for the motor symptoms of Parkinson's disease. Mov Disord. 2011;26(Suppl 3):S2-41.

50. Keus SHJ, Munneke M, Graziano M. European Physiotherapy Guideline for Parkinson's disease. KNGF/ParkinsonNet: The Netherlands; 2014.

51. Moher D, Liberati A, Tetzlaff J, Altman DG. Preferred reporting items for systematic reviews and meta-analyses: the PRISMA statement. PLoS Med. 2009:6:e1000097.

52. Heyn P, Abreu BC, Ottenbacher KJ. The effects of exercise training on elderly persons with cognitive impairment and dementia: a meta-analysis. Arch Phys Med Rehabil. 2004;85:1694-704

53. Review Manager (RevMan) [Computer Program]. The Cochrane Collaboration. Copenhagen: The Nordic Cochrane Centre; 2014.

54. Lim I, van Wegen E, de Goede C, Deutekom M, Nieuwboer A, Willems A, Jones D, Rochester L, Kwakkel G. Effects of external rhythmical cueing on gait in patients with Parkinson's disease: a systematic review. Clin Rehabil. 2005;19:695-713.

55. Tooth LR, Ottenbacher KJ. The kappa statistic in rehabilitation research: an examination. Arch Phys Med Rehabil. 2004;85:1371-6.
56. Frazzitta G, Maestri R, Ghilardi MF, Riboldazzi G, Perini M, Bertotti G, Boveri $\mathrm{N}$, Buttini S, Lombino FL, Uccellini D, et al. Intensive rehabilitation increases BDNF serum levels in parkinsonian patients: a randomized study. Neurorehabil Neural Repair. 2014;28:163-8.

57. Sajatovic M, Ridgel AL, Walter EM, Tatsuoka CM, Colon-Zimmermann K, Ramsey RK, Welter E, Gunzler SA, Whitney CM, Walter BL. A randomized trial of individual versus group-format exercise and self-management in individuals with Parkinson's disease and comorbid depression. Patient Prefer Adherence. 2017;11:965-73.

58. Cohen J. Statistical Power Analysis for the Behavioral Sciences. 2nd ed. Hillsdale, NJ: Lawrence Erlbaum; 1988.

59. Cochrane Handbook for Systematic Reviews of Interventions Version 5.1.0 [updated March 2011]. The Cochrane Collaboration, 2011.

60. Fontanesi C, Kvint S, Frazzitta G, Bera R, Ferrazzoli D, Di Rocco A, Rebholz H, Friedman E, Pezzoli G, Quartarone A, et al. Intensive Rehabilitation Enhances Lymphocyte BDNF-TrkB Signaling in Patients With Parkinson's Disease. Neurorehabil Neural Repair. 2016;30:411-8.

61. Zoladz JA, Majerczak J, Zeligowska E, Mencel J, Jaskolski A, Jaskolska A, Marusiak J. Moderate-intensity interval training increases serum brainderived neurotrophic factor level and decreases inflammation in Parkinson's disease patients. J Physiol Pharmacol. 2014;65:441-8.

62. Marusiak J, Zeligowska E, Mencel J, Kisiel-Sajewicz K, Majerczak J, Zoladz JA, Jaskolski A, Jaskolska A. Interval training-induced alleviation of rigidity and hypertonia in patients with Parkinson's disease is accompanied by increased basal serum brain-derived neurotrophic factor. J Rehabil Med. 2015:47:372-5.

63. Angelucci F, Piermaria J, Gelfo F, Shofany J, Tramontano M, Fiore M, Caltagirone C, Peppe A. The effects of motor rehabilitation training on clinical symptoms and serum BDNF levels in Parkinson's disease subjects. Can J Physiol Pharmacol. 2016;94:455-61.

64. Archer T. Influence of physical exercise on traumatic brain injury deficits: scaffolding effect. Neurotox Res. 2012;21:418-34.

65. El-Tamawy MS, Abd-Allah F, Ahmed SM, Darwish MH, Khalifa HA. Aerobic exercises enhance cognitive functions and brain derived neurotrophic factor in ischemic stroke patients. NeuroRehabilitation. 2014;34:209-13.

66. Lahera G, Bayon C, Fe Bravo-Ortiz M, Rodriguez-Vega B, Barbeito S, Saenz M, Avedillo C, Villanueva R, Ugarte A, Gonzalez-Pinto A, de Dios C. Mindfulnessbased cognitive therapy versus psychoeducational intervention in bipolar outpatients with sub-threshold depressive symptoms: a randomized controlled trial. BMC Psychiatry. 2014;14:215.

67. Horvath K, Aschermann Z, Acs P, Deli G, Janszky J, Komoly S, Balazs E, Takacs K, Karadi K, Kovacs N. Minimal clinically important difference on the Motor Examination part of MDS-UPDRS. Parkinsonism Relat Disord. 2015;21: 1421-6.

68. Molendijk ML, Spinhoven P, Polak M, Bus BA, Penninx BW, Elzinga BM. Serum BDNF concentrations as peripheral manifestations of depression: evidence from a systematic review and meta-analyses on 179 associations (N=9484). Mol Psychiatry. 2014;19:791-800.

69. Polacchini A, Metelli G, Francavilla R, Baj G, Florean M, Mascaretti LG, Tongiorgi E. A method for reproducible measurements of serum BDNF: comparison of the performance of six commercial assays. Sci Rep. 2015;5:17989.

70. Bus BA, Molendijk ML, Penninx BJ, Buitelaar JK, Kenis G, Prickaerts J, Elzinga BM, Voshaar RC. Determinants of serum brain-derived neurotrophic factor. Psychoneuroendocrinology. 2011;36:228-39.

71. Katoh-Semba R, Wakako R, Komori T, Shigemi H, Miyazaki N, Ito H, Kumagai T, Tsuzuki M, Shigemi K, Yoshida F, Nakayama A. Age-related changes in BDNF protein levels in human serum: differences between autism cases and normal controls. Int J Dev Neurosci. 2007;25:367-72.

72. El-Gharbawy AH, Adler-Wailes DC, Mirch MC, Theim KR, Ranzenhofer L, Tanofsky-Kraff M, Yanovski JA. Serum brain-derived neurotrophic factor concentrations in lean and overweight children and adolescents. J Clin Endocrinol Metab. 2006;91:3548-52.

73. Hashimoto K, Iwata Y, Nakamura K, Tsujii M, Tsuchiya KJ, Sekine Y, Suzuki K, Minabe $Y$, Takei N, lyo M, Mori N. Reduced serum levels of brain-derived neurotrophic factor in adult male patients with autism. Prog Neuropsychopharmacol Biol Psychiatry. 2006;30:1529-31.

74. Fernandes BS, Gama CS, Cereser KM, Yatham LN, Fries GR, Colpo G, de Lucena D, Kunz M, Gomes FA, Kapczinski F. Brain-derived neurotrophic factor as a state-marker of mood episodes in bipolar disorders: a systematic review and meta-regression analysis. J Psychiatr Res. 2011;45:995-1004.

75. Bocchio-Chiavetto L, Bagnardi V, Zanardini R, Molteni R, Nielsen MG, Placentino A, Giovannini C, Rillosi L, Ventriglia M, Riva MA, Gennarelli M. 
Serum and plasma BDNF levels in major depression: a replication study and meta-analyses. World J Biol Psychiatry. 2010;11:763-73.

76. Scalzo P, Kummer A, Bretas TL, Cardoso F, Teixeira AL. Serum levels of brainderived neurotrophic factor correlate with motor impairment in Parkinson's disease. Journal of Neurology. 2010;257:540-5.

77. Ventriglia M, Zanardini R, Bonomini C, Zanetti O, Volpe D, Pasqualetti P, Gennarelli M, Bocchio-Chiavetto L. Serum brain-derived neurotrophic factor levels in different neurological diseases. Biomed Research International. 2013. Article ID 901082. https://doi.org/10.1155/2013/901082.

78. Bos I, Jacobs L, Nawrot TS, de Geus B, Torfs R, Panis LI, Degraeuwe B, Meeusen R. No exercise-induced increase in serum BDNF after cycling near a major traffic road. Neuroscience Letters. 2011;500(2):129.

79. Bousquet M, Gibrat C, Saint-Pierre M, Julien C, Calon F, Cicchetti F. Modulation of brain-derived neurotrophic factor as a potential neuroprotective mechanism of action of omega-3 fatty acids in a parkinsonian animal model. Prog Neuropsychopharmacol Biol Psychiatry. 2009:33(9):1401-8.

80. He J, Xiang Z, Zhu X, Ai Z, Shen J, Huang T, Liu L, Ji W, Li T. Neuroprotective effects of 7, 8-dihydroxylflavone on midbrain dopaminergic neurons in MPP+-treated monkeys. Sci Rep. 2016 Oct 12; 6:34339. https://doi.org/10.1038/srep34339.

81. Van der Kolk NM, Speelman AD, van Nimwegen M, Kessels RP, IntHout Hakobjan M, Munneke M, Bloem BR, van de Warrenburg BP. BDNF polymorphism associates with decline in set shifting in Parkinson's disease. Neurobiol Aging. 2015;36:1605.e1601-e1606.

82. Mougeot JL, Hirsch MA, Stevens CB, Mougeot F. Oral biomarkers in exerciseinduced neuroplasticity in Parkinson's disease. Oral Dis. 2016;22:745-53.

83. Polacchini A, Metelli G, Francavilla R, Baj G, Florean M, Mascaretti LG, Tongiorgi E. A method for reproducible measurements of serum BDNF: comparison of the performance of six commercial assays. Scientific Reports. 2015;5:17989. https://doi.org/10.1038/srep17989.

84. Daviet JC, Roy X, Quelven-Bertin I, Jallageas R, Mandigout S, Torny F, Monteil J. Parkinson's patient runs an ultra marathon: a case report. Eur J Phys Rehabil Med. 2014;50:447-51.

85. Piepmeier AT, Etnier JL. Brain-derived neurotrophic factor (BDNF) as a potential mechanism of the effects of acute exercise on cognitive performance. Journal of Sport and Health Science. 2015;4(1):14-23.

86. Francardo V, Schmitz Y, Sulzer D, Cenci MA. Neuroprotection and neurorestoration as experimental therapeutics for Parkinson's disease. Experimental Neurology. 2017;298:137-47.

87. Rimmer JH, Chen MD, McCubbin JA, Drum C, Peterson J. Exercise intervention research on persons with disabilities: what we know and where we need to go. Am J Phys Med Rehabil. 2010;89:249-63.

88. Morris SB, DeShon RP. Combining effect size estimates in meta-analysis with repeated measures and independent-group designs. Psychological Methods. 2002; $7(1): \mathrm{N}-25$.

\section{Submit your next manuscript to BioMed Central and we will help you at every step:}

- We accept pre-submission inquiries

- Our selector tool helps you to find the most relevant journal

- We provide round the clock customer support

- Convenient online submission

- Thorough peer review

- Inclusion in PubMed and all major indexing services

- Maximum visibility for your research

Submit your manuscript at www.biomedcentral.com/submit

) Biomed Central 